\title{
Mantenedor de espacio removible: planificación del tratamiento y seguimiento de un caso clínico
}

Removable space maintainer: treatment planning and follow up of a clinical case
Manutenção de espaço removivel: planejamento do tratamento e acompanhamento de um caso clínico
Fecha de Recepción

23 de mayo 2019
Aceptado para su publicación

23 de junio 2019
María Lorena Cardoso

Ignacio Miguel Escobar

Daniela Yanina Burlli

Norma Griselda Díaz

Andrea Verónica Galiana

Asignatura Odontopediatría. Módulo Atención Integral del Niño y Adolescente.

Facultad de Odontología.

Universidad Nacional del Nordeste.

República Argentina.

\section{Autor de Correspondencia}

María Lorena Cardoso

Facultad de Odontología. UNNE. Av. Libertad 5450. Corrientes (Capital). República Argentina.

Email:marialorenacardoso@hotmail.com

\section{Resumen}

Es importante mantener la integridad de la dentición primaria hasta el momento de su exfoliación, puesto que juega un papel esencial en el desarrollo del niño y puede verse afectada por factores entre los cuales el más frecuente es la caries dental, pudiendo conducir a alteraciones en el desarrollo fisiológico, psicológico y social del niño. El objetivo de este trabajo es presentar el tratamiento de rehabilitación oral y el seguimiento realizado, a un paciente niño de 8 años y 5 meses de edad, con pérdida prematura de múltiples piezas dentarias primarias por caries. Se realizaron exodoncias, restauraciones, y el mantenedor removible. Las acciones realizadas para la confección de este último fueron: preparación del terreno en la cavidad oral, toma de impresión y obtención de modelos, diseño de las placas superior e inferior, envío al laboratorio; primera prueba: placa base y rodetes de cera, para determinar la dimensión vertical; segunda prueba: enfilado, finalmente prueba del mantenedor. Se obtuvo, el restablecimiento de las funciones dentales, con un cambio evidente en la personalidad del paciente.

Palabras Claves: Rehabilitación bucal, Prostodoncia, Odontología Pediátrica 


\section{Abstracs}

It is important to maintain the integrity of the primary dentition until its exfoliation. It plays an essential role in the development of the child and can be affected by different factors. Among them, the most frequent is dental caries, which can lead to alterations in the physiological, psychological and social development of the child. The objective of this work is to present the oral rehabilitation treatment and the observation performed to an 8-year-5-month male patient with premature loss of multiple primary dental teeth due to caries. Extractions and restorations were performed, and the removable maintainer (RM) was used. The actions carried out for the preparation of the RM were: site preparation of the oral cavity, dental impression taking to obtain the models, design of the upper and lower plates. Then, it was sent to the laboratory. First test: baseplate and wax rims to determine the vertical dimension, test of the line, test of the maintainer. The restoration of dental functions was obtained with an evident change in the personality of the patient.

Key Words: Mouth Rehabilitation, Prosthodontics, Pediatric Dentistry

\section{Resumo}

É importante manter a integridade da dentição decídua até a sua esfoliação, uma vez que desempenha um papel essencial no desenvolvimento da criança e pode ser afetada por fatores dentre os quais o mais frequente é a cárie dentária, o que pode levar a alterações no desenvolvimento fisiológico, psicológico e social da criança. $\bigcirc$ objetivo deste trabalho é apresentar o tratamento de reabilitação oral e $\circ$ acompanhamento efetuado para um paciente do sexo masculino de 8 anos e 5 meses com perda prematura de múltiplos dentes primários por cárie. Extrações, restaurações e o mantenedor removível foram realizados. As ações realizadas foram a preparação do solo na cavidade oral, tendo uma impressão e a obtenção de modelos, desenho das placas superiores e inferiores, as quais foram enviadas ao laboratório; primeiro teste: base de prova e plano de cera, para determinar a dimen- são vertical; segundo teste: teste da linha, finalmente teste do mantenedor. A restauração das funções dentárias foi obtida, com uma evidente mudança na personalidade do paciente.

Palavras-chave: Reabilitação Bucal, Prostodontia, Odontopediatria

\section{Introducción}

El tratamiento odontológico integral del paciente pediátrico debe realizarse bajo una planificación acorde con la edad y la complejidad del caso. Cuando el paciente presenta múltiples lesiones de caries con afección pulpar e imposibilidad de restauración, se hace necesaria la realización de exodoncias. Se considera pérdida prematura de dientes primarios cuando éstos se exfolian o son extraídos antes del momento fisiológico de recambio'. Sus consecuencias incluyen cambios en el plano sagital: pérdida de perímetro de arco y longitud de arco por migración mesial de los molares permanentes, y por distoinclinación o retrusión de los dientes anteriores, pérdida del espacio disponible para la correcta alineación de los dientes permanentes, lo cual producirá apiñamiento, erupción ectópica o impactación dentaria. En el plano vertical, producen profundización de la mordida y en el plano transversal, mordidas cruzadas ${ }^{2}$. Los mantenedores de espacio son aparatos ortodóncicos que pretenden impedir el cierre de espacio, seguido de la pérdida prematura de un diente primario. Se clasifican en fijos y removibles de acuerdo a su retención. Para su colocación deben ser tomados en cuenta ciertos factores como son: la edad a la que ocurrió la pérdida, tipo de diente perdido, presencia del diente sucesor, análisis del espacio disponible y tiempo transcurrido desde la pérdida. Su uso es indicado por la mayoría de los autores, ${ }^{3}$ aunque autores como Laing, Ram y Bijoor sugieren que en algunos casos puede ser innecesario su uso ${ }^{4-6}$.

El abandono en el que se encuentra la salud bucal de la población infantil, hace que los dientes de la primera dentición lleguen en un porcentaje mínimo al límite señalado por la naturaleza para su exfoliación fisiológica, convirtiéndose en una constante la aparición de 
REVISTA FACULTAD DE ODONTOLOGÍA

ISSN 1668-7280 / ISSN-E 2683-7986

42
CASO CLÍNICO

Cardoso - Escobar - Burlli - Diaz - Galiano caries, que avanzan hasta penetrar al tejido pulpar, culminando con la débil resistencia de este tejido ${ }^{7}$. En consecuencia, las complicaciones del periápice tan comunes en estos casos, obligan aún al profesional más conservador a proceder a la extracción de la pieza dentaria y en algunas ocasiones sin medir las consecuencias. El objetivo de este trabajo, es presentar el tratamiento de rehabilitación oral y el seguimiento realizado, a un paciente niño de 8 años y 5 meses de edad con pérdida prematura de múltiples piezas dentarias primarias por caries.

\section{Caso Clínico}

Se presentó a la Clínica de Odontopediatría un paciente de sexo masculino de 8 años y 5 meses de edad para su rehabilitación integral. Previa firma del consentimiento informado por parte del tutor, se procedió a realizar una completa historia clínica, en la que se hallaron los datos clínicos y radiográficos necesarios para la planificación del tratamiento rehabilitador adecuado para el paciente. Se realizaron las exodoncias y restauraciones necesarias, a fin de preparar la cavidad oral para recibir el mantenedor de espacio removible. Posteriormente se tomaron las impresiones, obteniéndose los modelos de trabajo sobre el que se diseñó el mantenedor de espacio, tanto superior como inferior, con tornillos medios para acompañar el crecimiento fisiológico del paciente, (Figura I). Después de las impresiones y la obtención del modelo de trabajo se confeccionaron las placas base, sobre estos se construyeron los rodetes de cera a fin de determinar la dimensión vertical y la relación céntrica, (Figura 2 ). Se realizaron las marcaciones a través del registro de mordida sobre los rodetes. En el laboratorio dental se realizó el enfilado, probándose nuevamente en boca, (Figura 3). Por último, se envió al laboratorio para acrilar la prótesis, obteniéndose el mantenedor de espacio terminado, (Figura 4).

Finalmente fue instalado el dispositivo protético, comprometiendo al paciente a controles mensuales para realizar los ajustes necesarios, a fin de acompañar el crecimiento fisiológico y realizar las modificaciones regulares, conforme erupcionen las piezas dentarias permanentes,
(Figura 5). Los cuales fueron realizados con dicha periodicidad, desde su colocación hasta la fecha, corroborando su correcto uso.

\section{Discusión}

Es necesario intervenir de manera temprana y adecuada sobre la dentición primaria para prevenir la caries, o tratar de forma conservadora los dientes con lesiones de caries, para evitar el desarrollo de una maloclusión; en aquellos casos donde los dientes se hayan perdido prematuramente, el uso de mantenedores de espacio es indispensable para preservar la integridad del perímetro del arco dental, de las relaciones oclusales y de la dimensión vertical ${ }^{8}$.

En la dentición primaria y mixta temprana, los molares primarios establecen las relaciones oclusales en los planos sagital, vertical y transversal, manteniendo el espacio para la erupción de los dientes permanentes. Los factores que determinan el efecto de las pérdidas prematuras sobre la oclusión son: las características previas existentes en la arcada dentaria, edad a la cual ocurrió la pérdida y la pieza dentaria primaria que se pierde?.

Las secuelas de las pérdidas prematuras incluyen cambios en el plano sagital, pérdida de perímetro y longitud de arco, con la consecuente pérdida de espacio disponible para la alineación de los dientes permanentes que produce apiñamiento, erupción ectópica o impactación de los mismos. Esto ocurre por migración mesial de los molares permanentes, y por distoinclinación o retrusión de los dientes del segmento anterior. Se han descrito alteraciones en las relaciones molar y canina debido a estas migraciones dentarias ${ }^{10}$. Como también, cambios en el plano vertical, como profundización de la sobremordida y en el plano transversal, se han observado mordidas cruzadas por el sentido de la migración dental'".

La evidencia permite inferir que, en algunas situaciones clínicas, las extracciones de molares primarios, sin el tratamiento preventivo adecuado, provocarían pérdida de espacio y alteraciones de las relaciones sagitales ${ }^{12}$. La migración dental que ocurre incluye la mesialización de molares $y$, en algunos casos, la distalización 
de caninos primarios inferiores. Esta migración dental determina, además del acortamiento del perímetro de arco, la alteración de la relación molar y canina.

En los trabajos realizados por varios autores en donde se asocian la pérdida prematura de molares deciduos y el establecimiento de una mordida cruzada anterior, se observa que a medida que se pierden más dientes y la brecha edéntula es más grande, los efectos encontrados son más perjudiciales y con mayor predisposición a la mordida cruzada anterior ${ }^{13}$. Esto puede deberse a que, no sólo hay acortamiento del perímetro de arco, sino que hay alteraciones en los tres planos espaciales ocasionadas por la falla en el soporte posterior de la oclusión, disminuyendo la dimensión vertical y favoreciendo la propulsión mandibular en busca de mayor contacto oclusal.

Las prótesis parciales removibles son parte de un tratamiento prostodóntico integral, usadas en pacientes niños, de acuerdo con su desarrollo psicoemocional y neuromotor ${ }^{14}$. Este tipo de restauración generalmente se confecciona cuando existe pérdida prematura de piezas dentarias primarias y el germen de re- emplazo está en estadíos anteriores de 7.5 u 8 de Nolla ${ }^{15}$.

En otros trabajos se menciona que, la mayor cantidad de exodoncias de los dientes deciduos ocurren cuando sus respectivos dientes sucesores se encontraron en el estadío de Nolla 5 , lo que prioriza aún más la utilización de mantenedores de espacio a fin de minimizar alteraciones oclusales ${ }^{16}$.

Estas prótesis parciales removibles deben preservar las piezas remanentes y tejidos de sostén sin interferir con el crecimiento y desarrollo de las arcadas dentarias, mientras restituye la masticación y estética del paciente, así como también, restablecer una nueva relación oclusal y en otras ocasiones acondicionar dientes y tejidos de los rebordes con el objeto de obtener el máximo soporte para la prótesis definitiva $\circ$ un aparato correctivo ${ }^{17,18}$.

El tratamiento recibido por el paciente del caso presentado, permitió que se restablecieran sus funciones dentarias y de la cavidad bucal, permitiendo su normal desarrollo psicológico y social, luego de la utilización de las prótesis, observándose un cambio evidente en la personalidad del paciente.

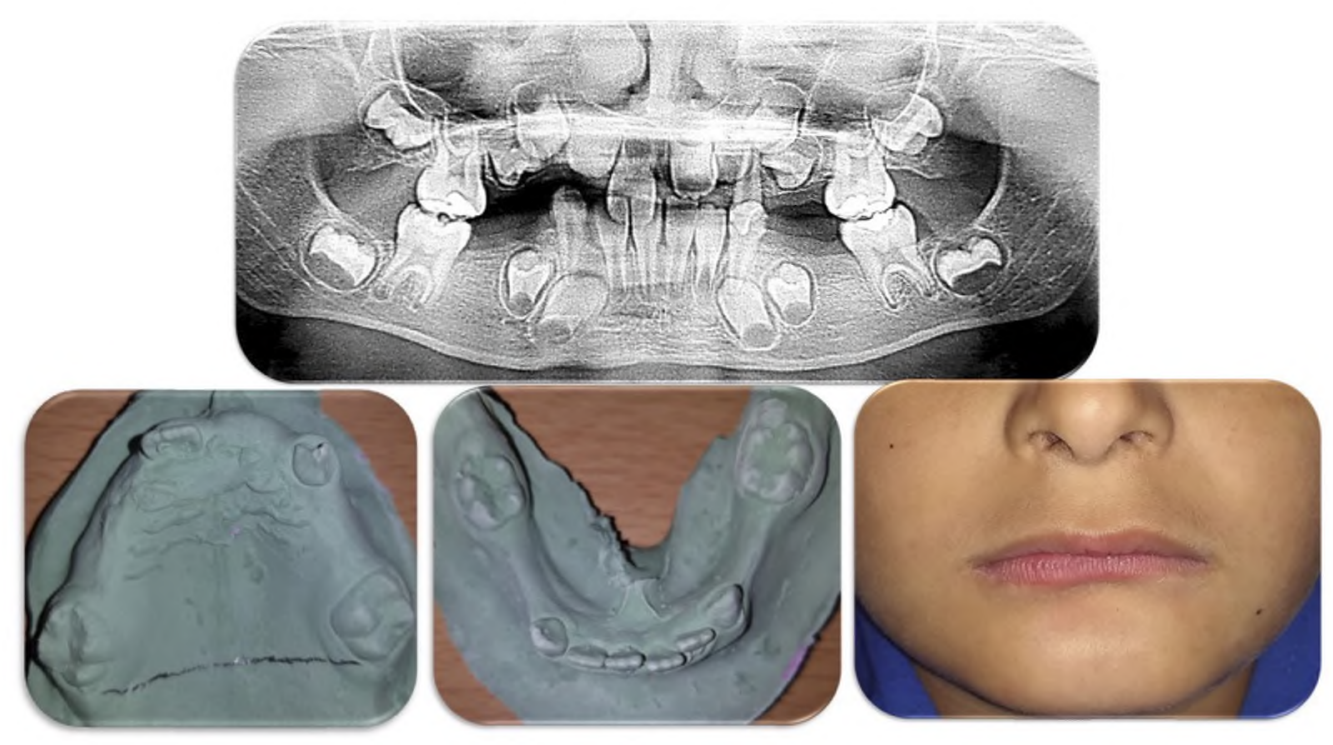

Figura 1. Fotografias representativas del estudio clínico y radiográfico realizado previo a la elaboración del plan rehabilitador. 
REVISTA FACULTAD DE ODONTOLOGÍA SSN 1668-7280 / ISSN-E 2683-7986

$44 \quad$ http://dx.doi.org/10.30972/fo. 1213873
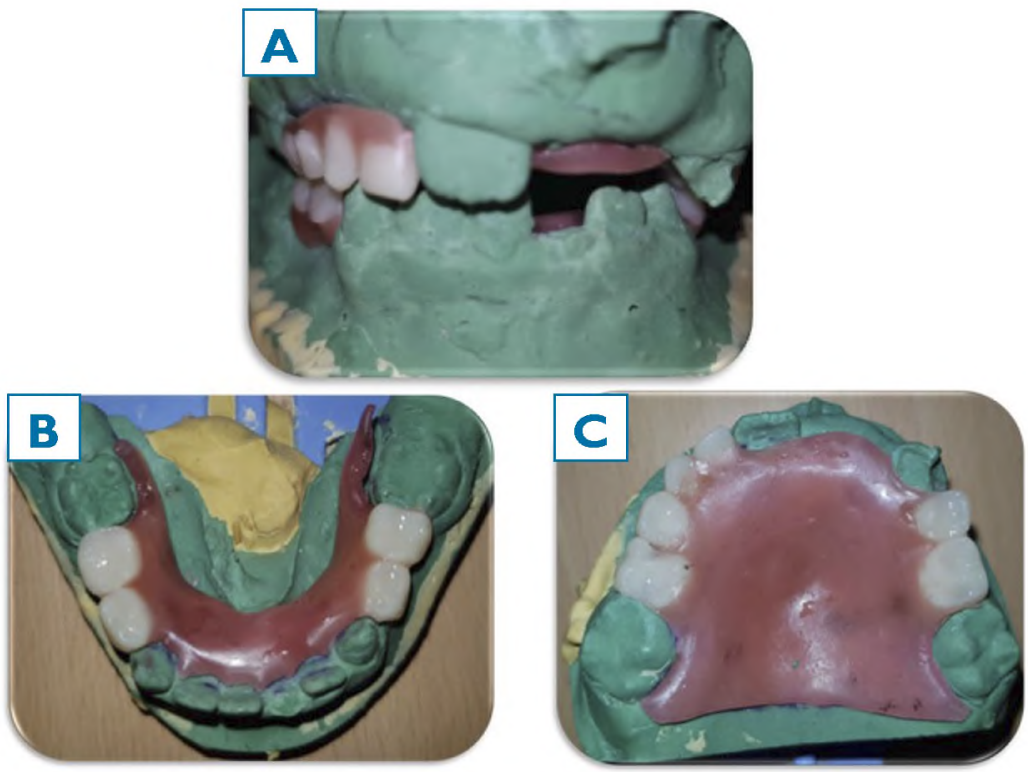

Figura 2. A y B. Fotografias representativas de las placas base y los rodetes de cera confeccionados. C. Determinación de la dimensión vertical y la relación céntrica a través de la indentación en los rodetes de cera.

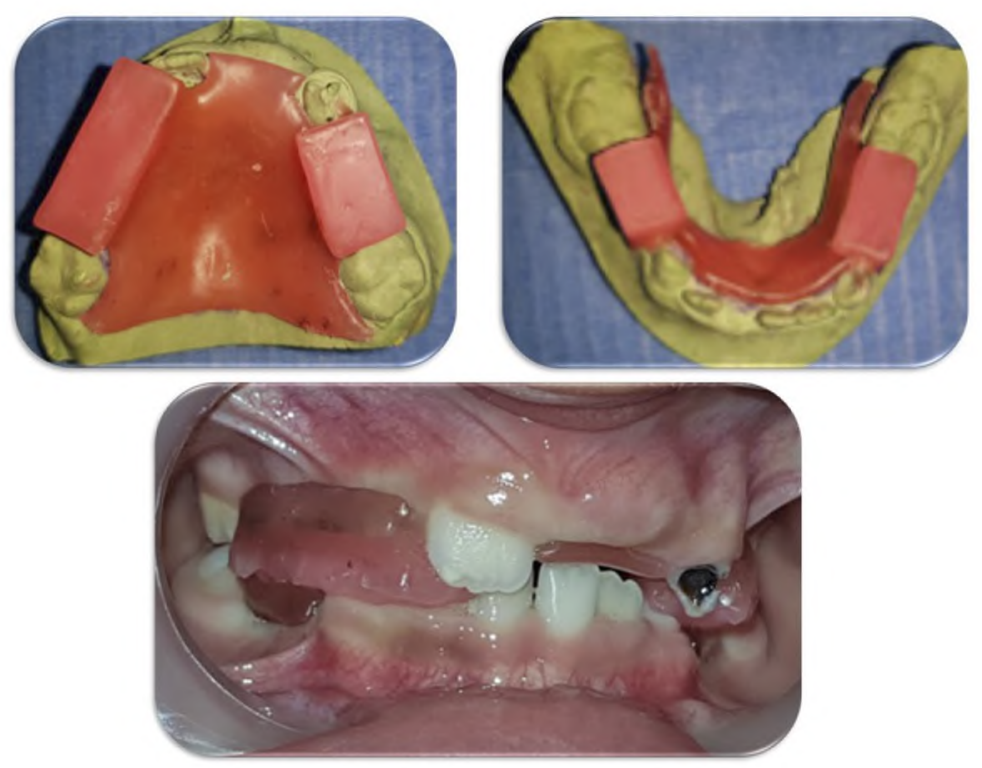

Figura 3. Fotografias de los enfilados realizados en los modelos de estudios. 


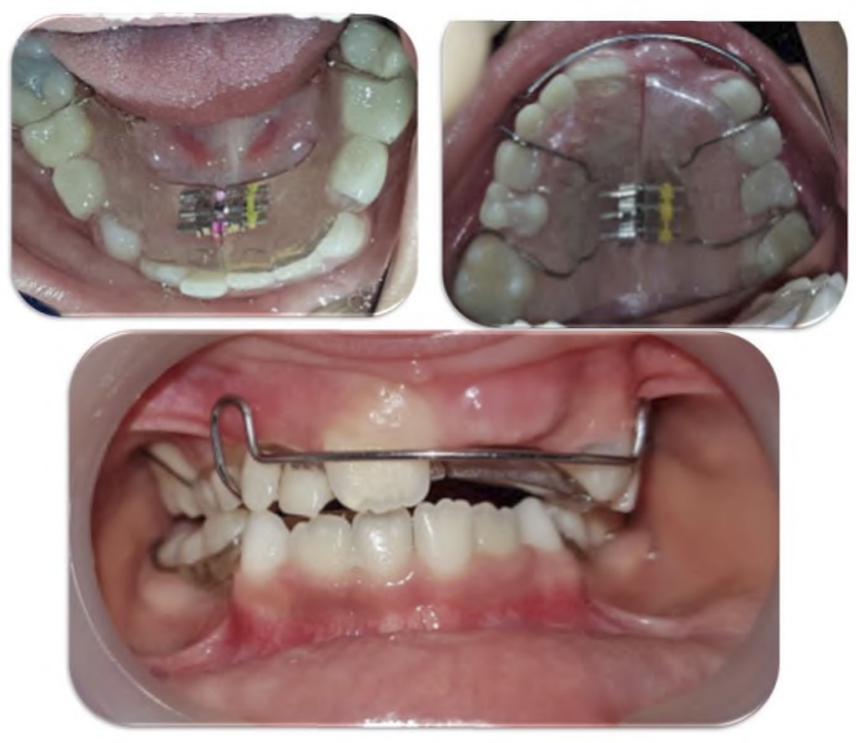

Figura 4. Fotografia de la instalación del mantenedor removible, nótese la correcta adaptación del acrílico sin interferir con el crecimiento fisiológico.
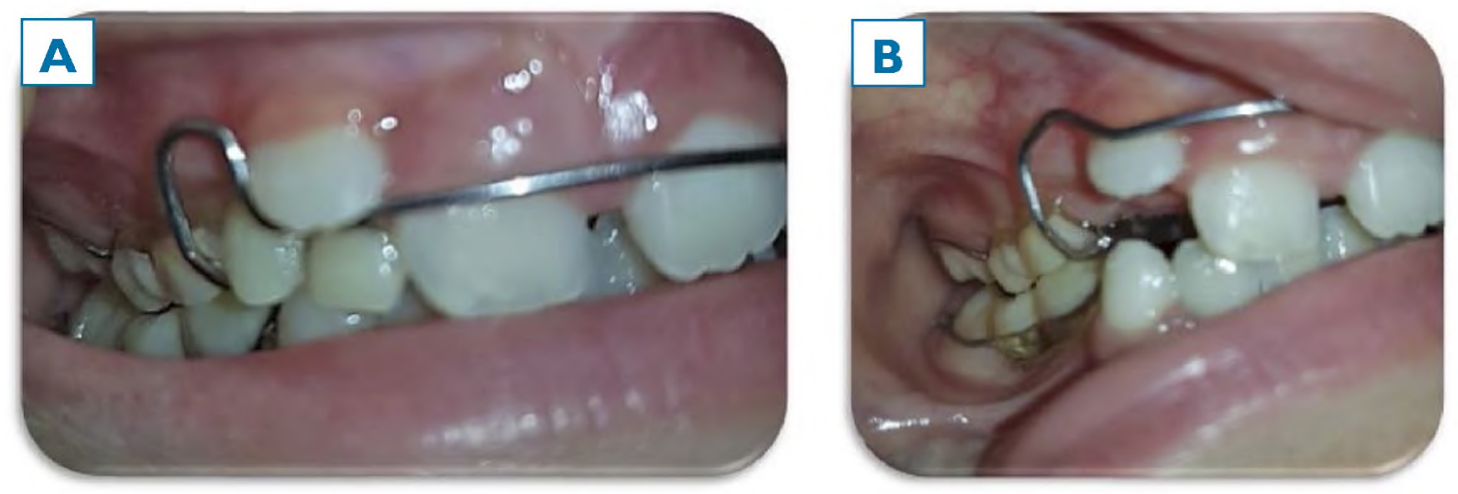

Figura 5. A. Fotografia de uno de los controles de ajuste en el que incluyo un aumento de $A \square$ de vuelta al tornillo medio de ambos maxilares acompañando el crecimiento y la correspondiente adaptación del sector superior lateral derecho para permitir la erupción de la pieza 2.2. B. La adaptación consistió en la remoción de las piezas de stock y del acrílico con la correspondiente adaptación del arco vestibular para guiar la erupción de la pieza dentaria. 
REVISTA FACULTAD DE ODONTOLOGÍA ISSN 1668-7280 / ISSN-E 2683-7986

46
CASO CLÍNICO

Cardoso - Escobar - Burlli - Diaz - Galiana

\section{Referencias Bibliográficas}

I. Guideline on dental management of heritable dental developmental anomalies. Pediatr Dent. 2013; 35 (5): EI 79-EI84.

2. García Y, Da Silva L, Medina A, Crespo $\bigcirc$. Efecto de la pérdida prematura de molares primarios sobre la relación horizontal incisiva. Rev. Odontopediatr Latinoam. 20I I; I (I): 49-57.

3. Law CS. Management of premature primary tooth loss in the child patient. J Calif Dent Assoc. 20I3; 4I (8): 6I2-8.

4. Bijoor R, Kohli K. Comtemporary Space Maintenance. J Paediatr Dent. 2005; 7I (2): 32-35.

5. Laing E, Ashley P, Naini F, Gill D. Space maintenance. Int J Paediatr Dent. 2009; 19 (3): I55-|62.

6. Ram D, Ben M, Efrat J, Moskovitz M. Mantenedores de espacio mandibulares para la prevención de pérdida de espacio, posterior a la pérdida prematura de molares primarios. Odontol Pediatr. 20I0; 9 (2): I27-| 40.

7. Cardoso M. Escobar I. Acosta J. Burlli D. Diaz N. Rehabilitación oral en bebes, enfoque preventivo y psicológico. Odontol Pediatr. 2018; 17 (I): 70-78.

8. Medina A, Crespo O, Da Silva L. Factores de riesgo asociados a maloclusión en pacientes pediátricos. Acta Odont Venez. 2010; 48 (2): I-I3.

9. Tunison $W$, Flores-Mir C, ElBadrawy $H$, Nassar U, El-Bialy T. Dental arch space changes following premature loss of primary first molars: a systematic review. Pediatr Dent. 2008; 30 (4): 297-302.

10. Al-Shahrani N. The prevalence of premature loss of primary teeth and its impact on malocclusion in the Eastern Province of Saudi Arabia. Acta Odontol Scand. 2015; 73 (7): 544-9.

I I. García S. Space maintainer in the prevention of malocclusions: a case report. Arch Méd de Camagüey. 2014; I8 (2): 193-199.

12. Cernei E, Maxim D, Zetu I. The influence of premature loss of temporary upper molars on permanent molars. Rev Med Chir Soc Med Nat. 20I5; I 19 (I): 236-42.
13. Garcia YM, Da Silva de Carballo LA, Medina CA Crespo O. Efecto de la pérdida prematura de molares primarios sobre la relación horizontal incisiva. Rev ALOP. 201 I. https:// www.revistaodontopediatria.org/ediciones/20I I/I/art-8I.

14. Guedes-Pinto, A. (2003). Recursos protésicos en Odontopediatria. En S. L. Ferreira, H. Biancalana \& A.C. Guedes-Pinto (Comp.) Rehabilitación bucal en odontopediatria. Atención integral. Colombia: Amolca; 2003.

15. Nolla CM. The Development of the Permanent Teeth. Journ Dent Child. 1960. 254266. Cardoso MI, Falcon DE, Galiana AV. Opciones de tratamiento en un paciente con pérdida prematura de segundos molares primarios: zapato distal modificado. UnivOdontologica. 20I 7;35 (75). http://revistas. javeriana.edu.co/index.php/revUnivOdontologica/article/view/ $|8| 26$ ).

16. Huaman Ricce LL. Pérdida prematura de dientes deciduos en niños de 3-9 años de edad sometidos a tratamiento odontológico integral bajo anestesia general en el Instituto Nacional de Salud del Niño, 2014. http://cybertesis.unmsm.edu.pe/handle/ cybertesis/397I?show=full

17. López-Gómez SA, Villalobos-Rodelo JJ, Ávila-Burgos L, Casanova-Rosado JF, VallejosSánchez AA, Lucas-Rincón SE, Patiño Marín $N$, Medina-Solís CE. Relation-ship between premature loss of primary teeth with oral hygiene, consumption of so drinks, dental care, and previous caries experience. Sci Rep. 2016;6:2। |47. doi: 10.1038/srep2 I |47.

18. American Academy of Pediatric Dentistry and the American Academy of Pediatrics. Policy on Early Childhood Caries (ECC): Classifications, Consequences, and Preventive Strategies. Pediatr Dent 2016. https:// www. aapd.org/media/policies_guidelines/P_ eccclassifications.pdf. 